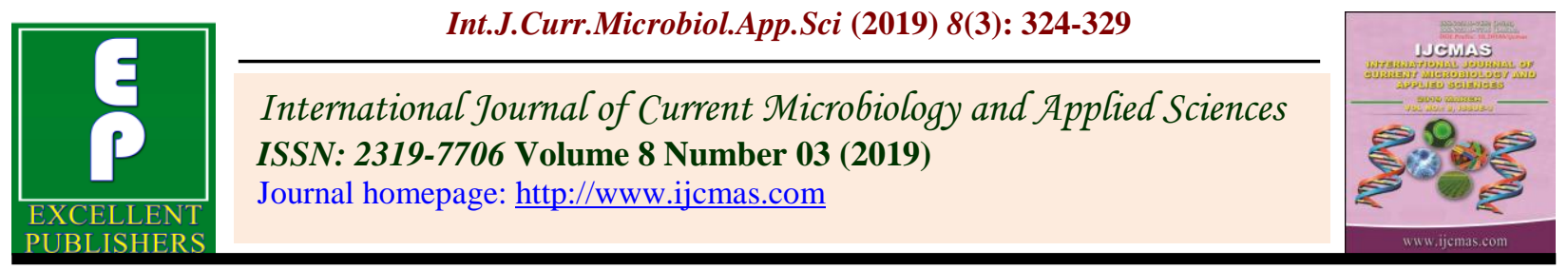

Original Research Article

https://doi.org/10.20546/ijcmas.2019.803.040

\title{
A Comparative Study on CD4 Count and Sputum Smear Examination by Fluorescent Microscopy in Retroviral Positive Patients in a Tertiary Care Centre
}

\author{
M.A. Ashiha Begum*, Kumar and Mani \\ Kanyakumari Govt. Medical College and Hospital, Kanyakumari, Tamilnadu, India \\ *Corresponding author:
}

\section{Keywords}

TB HIV, PLWHA, HIV testing, Sputum microscopy, CD4 count

Article Info

Accepted: 04 February 2019 Available Online: 10 March 2019

\section{A B S T R A C T}

TB is the most common opportunistic infection (OI) among HIV infected individuals, and co infected individuals are at high risk of death. TB is the largest single cause of death in the setting of AIDS, accounting for 26\% of AIDS related deaths, $99 \%$ of which occur in developing countries. HIV Associated Tuberculosis remains a major global public health challenge Hence routine TB screening among PLWHA (People Living with HIV-AIDS) offers the opportunity to identify those without TB, helps for early diagnosis and promptly treat TB. The aims of the study are 1 . To know the prevalence of HIV/TB Co-infection in a Tertiary care centre in a rural area 2. To find the $\mathrm{CD}_{4}$ count which gives Sputum smear positivity and negativity. Through Sputum smear examination by Fluorescent microscopy, routine HIV Testing by Rapid Test methods and $\mathrm{CD}_{4}$ count by Flowcytometry method are planned for this prospective study. In this study, out of $65 \mathrm{HIV}$ positive Patients 30 (46\%) had TB HIV coinfection, were started on Antituberculous treatment and remaining 35 (54\%) were retroviral positive only. Prevalence of HIV/TB co-infection is $46 \%$ among the sample size in this study. Prevalence is more in males and reproductive age group 16-45 years as $60 \%$ of patients fall into this group.

\section{Introduction}

Tuberculosis Immunodeficiency (TB) and Human Acquired Immunodeficiency Syndrome (HIVAIDS) constitute the main burden of infectious disease in developing countries ${ }^{1}$. Around 14 million individuals worldwide are estimated to be dually infected ${ }^{2,4}$. Most TB cases are in South East Asia, African and Western Pacific regions and an estimated 1113 per cent of incident cases were HIV
Positive $^{2}$. HIV-TB Co-infection most powerful risk factor for progression of $M$. tuberculosis infection. The two pathogens $M$. tuberculosis and HIV potentiate one another accelerating immunological deterioration ${ }^{3}$. TB may occur at any stage of HIV disease and is frequently the first recognized presentation of underlying HIV infection., ${ }^{2,5}$ The two pathogens M. tuberculosis and HIV potentiate one another accelerating immunological deterioration. Various lines of evidence indicate that inborn errors of immunity, as 
well as genetic polymorphisms, have an impact on susceptibility to TB and $\mathrm{HIV}^{5}$.

The risk of TB in HIV continues to increase as CD4 cell counts progressively decline ${ }^{6}$. As a result of WHO's 3 by 5 campaign, $>6$ million HIV infected individuals in resource limited settings have had access to antiretroviral therapy (ART) since 2004, which is for short of actual need, although ART can reduce the incidence of TB both at the individual and population level. PLWHA on ART still have higher TB incidence rates and a higher risk of dying from $\mathrm{TB}^{7}$, which may be due to delayed initiation of ART or the fact that patients present with advanced TB or both Routine TB screening among PLWHA offers ${ }^{10}$.

-The opportunity to identify those without TB

-Prevent TB by chemoprophylaxis

-Diagnose and promptly treat TB

\section{Materials and Methods}

This is a Cross-sectional study conducted by the Department of Microbiology and ART, Govt. TVR Medical College and Hospital. After obtaining the Institutional Ethical committee approval, the study was conducted from January 2014 to August 2014.About 208 patients who attended the Integrated Counseling and Testing Centre were included in the study, of them only 65 were retroviral positive and they were subjected to the sputum smear examination by Fluorescent microscopy and $\mathrm{CD}_{4}$ count.

\section{HIV testing method}

A total of 208 patients were screened for HIV by using WHO approved Elisa Rapid kits based on Immunoconcentration, Dot blot assay and Immunochromatography methods, 65 were found retroviral positive.

\section{$\mathrm{CD}_{4}$ counts}

Blood samples were collected after obtaining written informed consent $\mathrm{CD}_{4}$ counting done. Whole blood sample is collected from the 65 patients in EDTA liquid vacutainer tubes and the samples were processed on the same day using Fluorescence-activated cell sorting (FACS) COUNTER for determining the CD4 counts by the Flowcytometry method, (Fluorochrome labeled monoclonal antibodies to the $\mathrm{CD}_{4} \mathrm{~T}$ cells). Initially control run was done. Controls supplied with $\mathrm{CD}_{4}$ kit were prepared by adding normal blood and fixative solution to the $\mathrm{CD}_{4}$ reagent tube. Before running the reagent tubes on the FACS COUNTER control beads were added.

Patient samples were prepared by adding blood samples, fixative solution to the $\mathrm{CD}_{4}$ tube.

A reagent tube is taken, labeled and vortexed. Then tubes were cored and 50 microlitre of patient's blood added, vortexed again and incubated, Fixative solution added and vortexed. Samples were run in instrument and $\mathrm{CD}_{4}$ count results recorded

\section{Sputum microscopy}

Patients were asked to collect two sputum samples (1 early morning and 1 spot). Samples were labeled, smears were prepared from purulent part of the sputum and heat fixed.

- Staining was done using fluorescent stains

- $0.1 \%$ Auramine $\mathrm{O}$ was added and kept for 7 minutes

- Washed with water

- Decolourised with $0.5 \%$ acid alcohol for 2 minutes, washed with water

- Counterstained with $0.5 \%$ potassium permanganate for 30 seconds, washed and air dried

- Using LED fluorescence microscopy slides 
were examined at low power magnification$250 \mathrm{X}$ and $400 \mathrm{X}$, which allows larger area per unit of time for examination and this is 6 $\%$ more sensitive than light microscopy.

\section{Results and Discussion}

Among the 208 patients screened for HIV, 65 were retroviral positive and were screened for pulmonary tuberculosis by sputum smear for Acid Fast Bacilli (AFB) using fluorescent Microscopy and by chest $\mathrm{X}$ ray. Sputum smear positive for AFB received Directly Observed Therapy Short course (DOTS). The results were analysed using SPSS (version 13) with the level of significance $p=0.05$

Table.1 Statistical analysis

\begin{tabular}{|l|c|c|c|}
\hline Gender & $\begin{array}{c}\text { HIV reactive } \\
\text { Sputum negative } \\
\text { No \% }\end{array}$ & $\begin{array}{c}\text { HIV Reactive } \\
\text { Sputum Positive } \\
\text { No \% }\end{array}$ & Total \\
\hline Male & $2366 \%$ & $2067 \%$ & No \% \\
\hline Female & $1234 \%$ & $1033 \%$ & $2366 \%$ \\
\hline Total & $35100 \%$ & $30100 \%$ & $65100 \%$ \\
\hline
\end{tabular}

\section{HIV/TB Co-infection}

Of the 65 retroviral positive cases, 30 had
HIV/ TB co-infection, thus $46 \%$ of patients had dual infection. Co-relating with gender 20 (67\%) were males and $10(33 \%)$ were females.

Table.2 HIV/TBCO-Infection and age

\begin{tabular}{|l|c|c|c|}
\hline Age & Male & Female & Total \\
\hline $\mathbf{1 - 1 5}$ & 1 & 0 & $1(3.3 \%)$ \\
\hline $\mathbf{1 6 - 3 0}$ & 4 & 3 & $7(23.3 \%)$ \\
\hline $\mathbf{3 1 - 4 5}$ & 7 & 4 & $11(36.6 \%)$ \\
\hline $\mathbf{4 6 - 6 0}$ & 5 & 3 & $8(26.6 \%)$ \\
\hline $\mathbf{6 1 - 7 5}$ & 3 & 0 & $3(10 \%)$ \\
\hline TOTAL & 20 & 10 & $30(100 \%)$ \\
\hline
\end{tabular}

Table.3 HIV/TB and $\mathrm{CD}_{4}$ count

\begin{tabular}{|l|c|c|c|}
\hline CD $_{4}$ Count & HIV/TB Co-Infection & HIV Alone & Total \\
\hline$<\mathbf{5 0}$ & $13(43.3 \%)$ & $2(5.7 \%)$ & 15 \\
\hline $\mathbf{5 0 - 1 5 0}$ & $11(36.6 \%)$ & $9(25.7 \%)$ & 20 \\
\hline $\mathbf{1 5 1 - 2 5 0}$ & $2(6.6 \%)$ & $8(22.8 \%)$ & 10 \\
\hline $\mathbf{2 5 1 - 3 5 0}$ & $2(6.6 \%)$ & $2(5.7 \%)$ & 4 \\
\hline$>\mathbf{3 5 0}$ & $2(6.6 \%)$ & $14(40 \%)$ & 16 \\
\hline TOTAL & $30(100 \%)$ & $35(100 \%)$ & 65 \\
\hline
\end{tabular}


Table.4 Sputum Negativity and $\mathrm{CD}_{4}$ Count

\begin{tabular}{|c|c|c|c|c|}
\hline $\mathrm{CD}_{4}$ Count & $<50$ & $50-150$ & $151-250$ & $>250$ \\
\hline No. of Patients & 4 & 11 & 4 & 15 \\
\hline
\end{tabular}

Prevalence of HIV/TB co-infection, a global estimate shows around 5.1 million people infected with HIV and about half of them are co-infected with $\mathrm{TB}^{2}$. In our study out of 65 Retroviral positive patients, 30 (46\%) had HIV/TB co-infection and were started on DOTS and the remaining 35 (54\%) Retroviral positive alone. Our study correlates with a North Indian study done by Naren et al in New Delhi. As per his studies, in developing countries TB is the most common life threatening, opportunistic infection in patients with dual infection ${ }^{6}$. He narrates 35-65\% patients of PLHA having TB of any organ. The incidence of dual infection was reported to be very high (50\%) in Sub Saharal Africa compared to that of Asia. The rate of dual infection varies in different regions of India, found to be between 0.4 and $20.1 \%$ in North India, 3.2\% in South India two decades back which increase to $20.1 \%$ now. And this increase may be due to improvement in diagnostic methods to detect $\mathrm{TB}^{10}$.

\section{Dual infection and age group}

In our study, the dual infection is higher in the reproductive age group of 16-45 years, $60 \%$ of the co-infected belong to this age group.Similarly Sameer Singhal et al study in co-infection from Wardha showed prevalence of dual infection was higher 55(84\%) in the age group og 16-45years ${ }^{12,13}$.

\section{Dual Infection and $\mathrm{CD}_{4}$ count}

Among other OI's like Cryptococcal meningitis or toxoplasmosis which occur in very low $\mathrm{CD}_{4}$ count, $\mathrm{TB}$ is unique it occurs over a wide range of $\mathrm{CD}_{4}$ count $<300$ cells per microlitre $\mathrm{CD}_{4}$ count ${ }^{14}$. In our study of 30 dual infection patients 93.4 had $\mathrm{CD}_{4}$ counts below 350 cells per microlitre. In sputum negative and retroviral positive cases 16 patients had a high $\mathrm{CD}_{4}$ counts, indicating sputum negativity has positive co-relation with high $\mathrm{CD}_{4}$ counts ${ }^{15}$. This is similar to the study done by Purushottam et al in Prevalence of Pulmonary TB among HIV positive patients attending Antiretroviral Therapy Clinic $^{11}$.

Summary and conclusion of the study are as follows

Prevalence of HIV/TB co-infection is $46 \%$ among the sample size in this study.

Prevalence is more in reproductive age group $16-45$ years as $60 \%$ of patients fall into this group

Sputum positive PTB had positive correlation with low $\mathrm{CD}_{4}$ counts as $93.4 \%$ had $\mathrm{CD}_{4}$ counts $<350$ cells per microlitre

Sputum negative PTB had positive correlation with high $\mathrm{CD}_{4}$ counts

\section{Recommendations}

In a study from South India, the medium survival in HIV infected presenting with PTB and EPTB(Extra Pulmonary TB) were found 45 and 40 months respectively

Most of the EPTB is missed in resource limited settings. About $30 \%$ of TB in HIV 
extra pulmonary. A Battery of tests are available including molecular techniques like NAAT- CBNAAT, PCR. So in resource limited settings at least we can do Sputum smear microscopy, chest Xray, which are cost effective and CB NAAT which is rapid and advanced molecular method which helps in early diagnosis and treatment, reduce the community spread of TB morbidity and mortality.

In 2011, app. 5\% of all diagnosed TB cases in India came from ICTC's which proves to be excellent sites for active TB case finding. Though close synergy between TB and HIV / Aids control programs were launched for active place finding with more advanced and rapid diagnostic mythology like CBNAAT to evaluate the resistance patterns also have to be provided for early diagnosis and treatment in rural areas. So these points should also be considered to improve the active TB case finding in HIV patients and hence will improve the early diagnosis and treatment, thus will reduce the morbidity and mortality

\section{Acknowledgement}

Our sincere thanks are due to TB State Task force and RNTCP programe for accepting the Operational research topic and ART - ICTC team of TVR Tertiary Care Hospital.

\section{References}

Bennedsen J, Larsen SO (1966) Examination for tubercle bacilli by fluorescence microscopy. Scand J Respir Dis 47: 114-120.

Cao J (2008) Surveillance of HIV infection among inpatients with TB. Journal of Applied Preventive Medicine 14: 7173.

Corbett EL, Watt CJ, Walker N, Maher D, Williams BG, et al., (2003) The growing burden of tuberculosis: global trends and interactions with the HIV epidemic. Arch Intern Med 163: 10091021.

Friedland G, Churchyard GJ, Nardell E (2007) Tuberculosis and HIV coinfection: current state of knowledge and research priorities. J Infect Dis 196: Suppl 1S1-3.

Girardi E, Raviglione MC, Antonucci G, Godfrey-Faussett P, Ippolito G (2000) Impact of the HIV epidemic on the spread of other diseases: the case of tuberculosis. AIDS 14: Suppl 3S47-56.

$\mathrm{He} C$ (2008) Investigation of TB/HIV cioinfection in rural area. Journal of AIDS \& STD 14: 292-293.

Hermans S, Nasuuna E, van Leth F, Byhoff E, Schwarz M, et al., (2012) Implementation and effect of intensified case finding on diagnosis of tuberculosis in a large urban HIV clinic in Uganda: BMC Public Health 12: 674.

Hermans SM, Castelnuovo B, Katabira C, Mbidde P, Lange JM, et al., (2012) Integration of HIV and TB services results in improved TB treatment outcomes and earlier prioritized ART initiation in a large urban HIV clinic in Uganda. J Acquir Immune Defic Syndr 60: e29-35.

Jiang X, Lu H, Zhang Y, Zhou Z, Ye H, et al., (2008) A cross-sectional study of HIV and tuberculosis coinfection cases. South Med J 101: 914-917.

Lawn SD, Churchyard G (2009) Epidemiology of HIV-associated tuberculosis. Curr Opin HIV AIDS 4: 325-333.

Li S, Zhang N, JiN J, Liu H, Zheng J, et al., (2008) Analysing the Screening Results of HIV Infection Among Patients with Pulmonary Tuberculosis.

Lin G, Duan R, Liu M, Li H, Wei H (2006) Surveillance of HIV infection among TB patients. Chinese Prevention Medicine 7: 446-447. 
McShane H (2005) Co-infection with HIV and TB: double trouble. Int J STD AIDS 16: 95-100; quiz 101.

Minion J, Pai M, Ramsay A, Menzies D, Greenaway C (2011) Comparison of LED and conventional fluorescence microscopy for detection of acid fast bacilli in a low-incidence setting. PLoS ONE 6: e22495

Shafer RW, Edlin BR (1996) Tuberculosis in patients infected with human immunodeficiency virus: perspective on the past decade. Clin Infect Dis 22: 683-704.

Study on the detection rate of double infection among patients with AIDS and TB and its influencing factors. Modern Preventive Medicine 34: 4457-4459.

Tang Z, Huang S, Wei C, Du L, Xu X (2009) Current status and management of HIVTB co-infection. GuangXi Medical Journal 31: 240-241.

Wang L, Liu W, Wang Y, Wu Z (2010) HIV prevalence among pulmonary tuberculosis patients China. J Acquir Immune Defic Syndr 53: Suppl 1S6165.

Zhen X (2004) Primary analysis of HIV infection in patients with pulmonary tuberculos. Chinese Journal of AIDS \& STD 10: 354-35

\section{How to cite this article:}

Ashiha Begum, M.A., Kumar and Mani. 2019. A Comparative Study on CD4 Count and Sputum Smear Examination by Fluorescent Microscopy in Retroviral Positive Patients in a Tertiary Care Centre. Int.J.Curr.Microbiol.App.Sci. 8(03): 324-329.

doi: https://doi.org/10.20546/ijcmas.2019.803.040 